\section{CUIDADANÍA Y PARTICIPACIÓN EN EL ESPACIO ELECTRÓNICO EUROPEO}

\author{
Javier Echeverría \\ Alfonso Unceta \\ Sinnergiak Social Innovation, \\ Universidad del País Vasco \\ jecheverria@sinnergiak.org \\ alfonso.unceta@ehu.es
}

\section{CITIZENSHIP AND PARTICIPATION IN THE EUROPEAN ELECTRONIC SPACE}

\begin{abstract}
The Lisbon Agenda and e-Europe 2002 and 2005 plans aimed to promote the European information and knowledge society. Although public participation was not a priority in the first phase, since 2005, the EU has shifted its strategy, promoting the creation of the Single European Information Space and fostering citizens' electronic participation, making it sometimes depend on e-inclusion, sometimes on governance. The i2010 program was a step towards the use of the European electronic space for citizen participation. The recent Digital Agenda for Europe, however, does not seem to prioritize e-participation, although it maintains plans for the e-inclusion and e-government.
\end{abstract}

KEY WORDS: Information society; citizens participation; governance; e-inclusion; e-participation.

\section{LA CONSTRUCCIÓN DEL ESPACIO ELECTRÓNICO EUROPEO}

Las tecnologías de la información y la comunicación (TIC) han transformado radicalmente las comunicaciones y el acceso, distribución y almacenamiento de la información, conformando un sistema tecnológico que se ha expandido por los diversos paises y sectores sociales, y ha modificado profundamente las relaciones humanas, así como las actividades empresariales e institucionales. Hoy en día, buena parte de la vida social resulta impensable sin las TIC.

Manuel Castells (1996-98) y otros autores han resumido esta profunda transformación diciendo que algunas sociedades industriales han generado una nueva modalidad de sociedad, la sociedad de la información, que también puede ser denominada sociedad red, debido a que gran parte de las interacciones sociales se desarrollan en red, al estar soportadas por las redes telemáticas. Casi todos los países han impulsado el desarrollo de las TIC y han promovido programas para fomentar el desarrollo de sus respectivas sociedades de la información. La propia ONU
RESUMEN: La Agenda de Lisboa y los planes e-Europe 2002 y 2005 tuvieron la finalidad de impulsar la sociedad europea de la información y el conocimiento. En una primera fase, la participación ciudadana no fue una prioridad. A partir de 2005 la UE reorientó su estrategia, promovió la creación del Espacio Único Europeo de la Información y fomentó la participación electrónica de los ciudadanos, haciéndola depender a veces de la e-inclusión, otras veces de la gobernanza. El programa i2010 supuso un paso importante hacia la utilización del espacio electrónico europeo para la participación ciudadana. La reciente Agenda Digital para Europa, en cambio, no parece priorizar la e-participación, aunque mantiene planes de e-inclusión y e-gobierno.

PALABRAS CLAVE: Sociedad de la información; participación ciudadana; gobernanza; e-inclusión; e-participación.

recomendó a finales del siglo XX este tipo de acciones y en la primera década del siglo actual convocó la Cumbre Mundial de la Sociedad de la Información (Ginebra, 2003 y Túnez, 2005), en la que participaron prácticamente todos los países del mundo, así como representantes de sociedades internacionales, empresas transnacionales del sector TIC y representantes de la sociedad civil de los cinco continentes, dando lugar a un ejemplo canónico de lo que podriamos denominar participación ciudadana global. Como resultado, se acordaron unos principios básicos para impulsar la sociedad de la información en todo el mundo y también se acordó un plan de acción, en el que se potenciaba la participación ciudadana en la sociedad de la información, formulando el siguiente principio: "todas las personas, en todas partes, deben poder participar y no debe excluirse a nadie de las ventajas que ofrece la sociedad de la información"1.

Por su parte, al poner en marcha la Agenda de Lisboa (2000), la Unión Europea decidió impulsar la sociedad de la información en los diversos países, regiones y ciudades europeas, promoviendo los programas eEurope 2002 
y eEurope 2005, cuyo objetivo principal era la creación de una "Europa electrónica", es decir, la construcción en Internet de un entramado de redes telemáticas que conectaran a los ciudadanos, instituciones y empresas europeas entre sí, con el fin de impulsar una sociedad de la información para todos, como afirmaba el lema del programa eEurope 2002. Se pretendía conseguir que la UE fuera en 2010 el líder mundial en el desarrollo de la sociedad de la información y el conocimiento, objetivo que ya en 2005 se reveló como inalcanzable. La Comisión Europea rediseñó la estrategia europea, proponiendo la Agenda Renovada de Lisboa ${ }^{2}$ y el programa $12010^{3}$, que sustituyó a los planes eEurope y fijó nuevos objetivos para desarrollar la sociedad de la información en los diversos paises y regiones de la UE.

El plan i2010 ha supuesto cambios sustanciales, al establecer tres prioridades:

"La Comisión propone tres prioridades para las políticas europeas de sociedad de la información y medios de comunicación:

i) la construcción de un Espacio único Europeo de la Información que promueva un mercado interior abierto y competitivo para la sociedad de la información y los medios de comunicación;

ii) el refuerzo de la innovación y la inversión en la investigación sobre las TIC con el fin de fomentar el crecimiento y la creación de más empleos y de más de calidad;

iii) el logro de una sociedad europea de la información basada en la inclusión que fomenta el crecimiento y el empleo de una manera coherente con el desarrollo sostenible y que da la prioridad a la mejora de los servicios públicos y de la calidad de vida"4.

En este artículo nos ocuparemos brevemente del primer objetivo y más ampliamente del tercero, en el que se fundamentan las políticas europeas de e-participación ciudadana. La creación de un Espacio Único Europeo de la Información (Single European Information Space, SEIS) implica un cambio radical en relación a la estrategia previa. En los dos planes eEurope el objetivo principal consistió en promover la conexión a Internet. Los indicadores para medir el nivel de desarrollo de la sociedad de la información en Europa dependían de dicho objetivo. El nuevo programa i2010 apenas aludió a la red, a diferen- cia de los planes eEurope que le precedieron, en donde sociedad de la información e Internet eran usadas como expresiones sinónimas 5 . La Comisión Europea rectificó en 2005 ese error conceptual y señaló que para construir un espacio europeo de la información, además de impulsar el desarrollo de Internet, eran precisas, como mínimo, otras dos grandes acciones estratégicas: la convergencia entre Internet y otros medios de comunicación (telefonía móvil, televisión digital, otras tecnologías TIC) y la convergencia entre las legislaciones de los diversos Estados europeos. En el año 2000, cuando se acordó la Agenda de Lisboa, se pensaba ingenuamente que la iniciativa privada iba a construir el espacio electrónico europeo a la vista de las grandes expectativas de negocio que la expansión de Internet prometía. En los documentos oficiales de aquella época la telefonía móvil y la televisión digital apenas eran mencionadas, siendo así que, si hoy en día la UE lidera a nivel mundial algún sector TIC, se trata precisamente de la telefonía móvil. En 2000 se hablaba casi exclusivamente de Internet y de la iniciativa privada; cinco años después se ha reconocido oficialmente que hay que impulsar otras tecnologías TIC, no sólo los ordenadores e Internet. También se ha tomado conciencia de la necesidad de definir políticas públicas para impulsar el desarrollo de la sociedad de la información, sin dejar de promover el desarrollo del mercado digital europeo, como se le denomina en el reciente documento sobre la Agenda Digital Europea de 2010.

Este giro conceptual tiene gran importancia. Aunque no se renuncia al objetivo de crear un mercado común europeo en el espacio electrónico ni a que dicho mercado crezca e incremente su peso relativo a nivel global, el plan i2010 asumió que en dicho espacio electrónico también pueden desarrollarse servicios públicos y nuevas formas de participación ciudadana. Por nuestra parte, consideramos que entonces fue cuando empezó a introducirse el concepto de e-ciudadanía europea, como intentaremos mostrar en el tercer apartado. La Comisión Europea ya había señalado en 2002 la necesidad de generar un espacio europeo de investigación y otro de enseñanza superior, superando la concepción exclusivamente económica del espacio electrónico. A partir de 2005 impulsó decididamente el gobierno electrónico (e-government) y dentro de ese marco la e-administración, la e-participación y la creación de nuevos servicios públicos en sanidad, seguridad, educación e investigación. Ello supuso incrementar las dimensiones 
del espacio electrónico europeo, donde hasta entonces habían predominado las actividades económicas, empresariales y comerciales:

"Hacen falta políticas proactivas para dar respuesta a los cambios fundamentales experimentados por la tecnología. La convergencia digital exige la convergencia política y una voluntad de adaptar los marcos reguladores cuando resulte necesaria para que sean coherentes con la economía digital emergente" ${ }^{\prime \prime}$.

La construcción del espacio europeo de la información (SEIS) implica una operación política que requiere concertación entre los Estados e importantes cambios en las normativas y regulaciones de cada país. El marco legislativo para una televisión sin fronteras, por ejemplo, exigiria una coordinación de las políticas estatales, lo que no parece fácil de lograrse. En efecto, ello implica un cambio conceptual importante, puesto que los medios de comunicación tradicionales, como la televisión, habrian de incorporar una función adicional: proporcionar acceso y conexión al espacio europeo de información (SEIS), que todavía está en fase de construcción. Por otra parte, los Gobiernos estatales y la propia Comisión pasan a convertirse en promotores del SEIS, cuyo diseño y desarrollo ya no se deja exclusivamente en manos del sector privado:

\begin{abstract}
"Si Europa quiere aprovechar al máximo su potencial económico, es necesario un enfoque político proactivo para estimular la evolución favorable de los mercados y la promoción de la sociedad del conocimiento (p. ej.: formación permanente, creatividad e innovación), la protección del consumidor y una sociedad de la información europea a la vez sana y segura"'.
\end{abstract}

En consecuencia, la Comisión Europea llamó a los Estados miembros a rediseñar sus planes nacionales y regionales, adaptándolos a los nuevos objetivos que se pretendían alcanzar. En el documento de la Comisión Europea se insistía continuamente en la interoperabilidad entre las diversas plataformas y los distintos países. También se impulsaba la convergencia legislativa en cuestiones de propiedad intelectual.

En cuanto al segundo objetivo, la inclusión en la sociedad europea de la información se convirtió un uno de los valores sociales preponderantes:
"Reforzar la cohesión social, económica y territorial consiguiendo que los productos y servicios de TIC sean más accesibles, en particular en las regiones menos adelantadas, constituye un imperativo económico, social, ético y político. En la iniciativa i2010 se hace particular hincapié en la participación plena y en que todo el mundo adquiera unas competencias digitales básicas"8.

Por tanto, la participación ciudadana en el espacio electrónico está ligada en el programa i2010 a la creación de una sociedad inclusiva de la información, así como a los programas de gobierno electrónico. Veamos de qué manera la CE ha promovido la e-participación, en el marco de una sociedad inclusiva de la información.

\section{El programa 12010 y las politicas DE E-PARTICIPACIÓN EN EUROPA}

El rechazo del borrador de Constitución Europea en 2005 fue un factor determinante para que la Comisión Europea comenzara a impulsar políticas activas de participación ciudadana ${ }^{9}$. Los Eurobarómetros mostraron que la ciudadanía se distanciaba cada vez más de la política europea y la CE intentó modificar esa tendencia, promoviendo el gobierno electrónico (e-Government) y la participación electrónica (e-participation) como modo de reactivar el interés ciudadano por la construcción de la UE. Esta última fue una de las cinco prioridades del e-Government Plan y se explicitó en 2007, cuando la CE publicó el documento Iniciativa Europea i2010 para la inclusión digital: "participar en la sociedad de la información"m.

Cuando se impulsa la participación ciudadana suele pensarse en la participación política, es decir, en la participación de la ciudadanía en la toma de decisiones políticas por parte de las instituciones y de los representantes electos. De hecho, se habla de participación ciudadana cuando "la ciudadanía se involucra en los procesos de determinación, discusión y ejecución de las políticas públicas, y constituye un instrumento estratégico para el reforzamiento de la gobernabilidad"11. Así es como ha concebido la Comisión Europea la participación ciudadana en las políticas de I+D+i, habiendo promovido diferentes formas de participación cívica $^{12}$. Sin embargo, el documento titulado "Participar en la sociedad de la información" utiliza una noción de

ARBOR Vol. 188756 julio-agosto [2012] 725-732 ISSN: 0210-1963

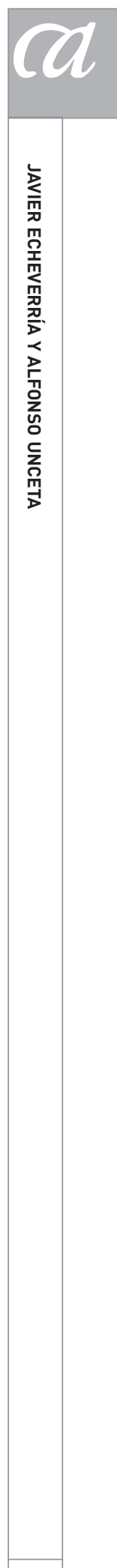

727 
participación distinta, que se centra en la participación social y económica, no en la participación política. Se afirma que existe un vínculo entre "el uso de las TIC y la participación económica y social de $\operatorname{todos}^{113} y$, más que incidir en las decisiones que toman las autoridades políticas, por ejemplo, la propia Comisión Europea, se apunta ante todo al objetivo de la inclusión en la sociedad de la información, del que se derivan varias formas de participación económica y social. Se pretende que todos los ciudadanos europeos puedan participar en la sociedad de la información, es decir, que dispongan de acceso, medios y capacidades para moverse en el espacio europeo de la información, independientemente de que una vez allí sus posturas sean proactivas o pasivas. Buena parte de la ciudadanía europea no es usuaria de las TIC $y$, por tanto, no puede participar en la sociedad de la información. Así pues, se trata de superar una barrera previa: conseguir que la gente esté en condiciones de poder participar, cosa que no está garantizada hoy en día, debido a la existencia de diversas brechas digitales en las sociedades europeas. No todas las TIC son accesibles a todos los europeos; hay grandes diferencias entre las competencias digitales de unos y otros; las escuelas están desigualmente dotadas, con lo que no garantizan la igualdad de oportunidades a

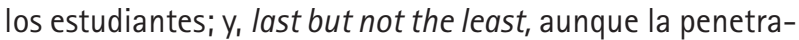
ción de Internet no para de aumentar, alrededor del 50\% de la población europea sigue sin utilizar regularmente sus servicios"14. En suma: la e-participación difiere del concepto usual de participación ciudadana porque está subordinada al logro de un objetivo previo, la inclusión digital, que es condición necesaria para poder integrarse en la sociedad de la información. La e-participación no tiene connotaciones políticas ni institucionales, sino que sus objetivos son impulsar las actividades económicas y las relaciones sociales en el espacio electrónico europeo, en el que se desarrolla la Europa electrónica (eEurope).

Partiendo de esta descripción de la situación, que, no hay que olvidarlo, se refiere al año 2007, el objetivo prioritario es la inclusión en la sociedad de la información, es decir, proporcionar los medios a toda la ciudadanía europea para operar con el sistema tecnológico TIC, en la medida en que éste da acceso a un nuevo espacio económico y social, el tercer entorno, cuyo desarrollo es considerado prioritario por parte de la Unión Europea ${ }^{15}$. Aunque los esfuerzos para garantizar la accesibilidad al espacio electrónico a los discapacitados fueron considerables en los programas
eEurope, y aunque la Unión Europea sea líder mundial al respecto, todavía queda mucho por hacer para garantizar el acceso y el uso competente de las TIC a las personas con diversidad funcional. Otro tanto cabe decir de los ancianos, de las personas que viven en ámbitos rurales y de muchas personas con pocos recursos económicos y escasa formación. Por lo general, estos sectores sociales están excluidos de la sociedad europea de la información y ni siquiera pueden desplegar formas de participación económica y social, mucho menos de e-participación política. La inclusión digital fue el objetivo principal del programa i2010, habiéndose formulado hitos concretos a alcanzar en la Declaración Ministerial de Riga (2006), buena parte de los cuales no han sido logrados. Dichos objetivos tenían que haberse satisfecho a finales de $2010^{16}$, pero el documento de 2007 alertaba ya que "de proseguir las tendencias actuales, será muy difícil el cumplimiento de la mayoría de esos objetivos a nivel comunitario"17. De hecho, la Comisión Europea ha constatado en 2010 que todavia hay un $30 \%$ de europeos que no ha usado nunca Internet ${ }^{18}$, dato que relativiza considerablemente el éxito de los programas eEurope, e incluso del programa $i 2010$.

Habia que modificar la estrategia en pro de la e-inclusión y la e-participación y por eso se encargó Study and Supply of Services on the Development of eParticipation in the EU, que fue elaborado para la Comisión Europea por tres centros de investigación: el Danish Technological Institute, la University of Leeds y la University of Macedonia. Dicho estudio ha sido publicado a finales de 2008 y luego reeditado a finales de 2009. Su elaboración ha estado precedida por diversas reuniones y simposios, por lo que existen materiales interesantes para analizar a fondo los diversos modos de concebir la e-participación en Europa. Aquí sólo vamos a referirnos a las conclusiones finales de dicho estudio, debido a que hay un giro importante en relación a los programas eEurope e i2010: la e-participación pasa a depender de la gobernanza europea, y con ello se acerca al concepto tradicional de participación ciudadana ${ }^{19}$. La ratificación del Tratado de Lisboa de 2007 ha sido, sin duda, un factor importante para dar este paso ${ }^{20}$. Las redes telemáticas y las TIC se convierten ahora en instrumentos para que los ciudadanos europeos puedan participar en los procesos de toma de decisiones por parte de la Comisión y del Parlamento Europeo. En suma, se trata de impulsar la participación democrática en el Single European Information Space (SEIS), cuya construcción se impulsa paralelamente. 
¿Cómo promover estas políticas de e-participación? Los autores de este Study propusieron un marco analítico en el que se distinguen tres niveles para impulsarla: un nivel básico (operacional), en el que se construyen herramientas y métodos para la e-participación; otro intermedio, con acciones y objetivos especificos, en el que los participantes obtienen beneficios para sí mismos gracias al uso exitoso de las herramientas anteriores; $y$ un tercero de alto nivel, en el que se producen impactos a nivel general, es decir, cuando se logran satisfacer objetivos sociales y valores públicos gracias a las acciones de e-participación previamente diseñadas. Dicho marco ha sido utilizado a fondo por tres de los autores del estudio, Smith, Macintosh y Millard (2009) y está basado en la distinción entre cinco componentes que hay que estudiar en cualquier iniciativa de e-participación:

1. Recursos: Infraestructuras TIC, tecnologías emergentes (web 2.0, minería de datos, procesamiento del lenguaje natural, servicios en la web semántica, entornos colaborativos, etc.), recursos humanos, recursos organizativos (liderazgo, gestión, equipos), financiación, tiempo requerido, etc.

2. Outputs operacionales: de índole tecnológica (hardware, software, canales, terminales...), herramientas de e-participación (solicitudes electrónicas, consultas electrónicas, voto electrónico, toma de decisiones, juegos, etc.), outputs organizativos (procedimientos para trabajar, reingeniería, formación del staff, cambios organizativos, etc.) y resultados derivados de la e-participación (estudios, análisis, etc.).

3. Beneficios derivados de la e-participación, tanto para los promotores del proyecto (reducción de costes, racionalización de los recursos, mayor productividad y eficiencia, aprendizaje por parte del staff, etc.), como para los usuarios (acceso, utilización de herramientas, cambios de patrones en los comportamientos, satisfacción creciente, empoderamiento, etc.) y para el conjunto de las personas e instituciones involucradas (stakeholders), por ejemplo: ahorro de tiempo, procedimientos más sencillos, mayor seguridad, menos burocracia, mayor transparencia, mejor implementación de las políticas, avances en la legislación, etc.

4. Impactos sociales, que a su vez son de diversas índole: ciudadanía activa, transparencia y apertura institucional, construcción de la esfera pública europea, fomento de la cultura basada en la consulta y el diálogo, subsidiariedad, empoderamiento de las comunidades y de las personas, inclusión, etc.

5. Factores externos al proyecto que han de ser tenidos en cuenta: tipo de gobernanza, tradiciones culturales de las agencias públicas, entorno legal, políticas de acompañamiento, autonomía, infraestructuras disponibles, entorno socio-económico y socio-cultural, etc.

Aun siendo mejorable, este marco analítico tiene la ventaja de ser lo suficientemente comprehensivo como para permitir el estudio de muy diversas formas de e-participación, distinguiendo tres niveles y cinco tipo de resultados, impactos y consecuencias. Resulta, por tanto, un avance considerable en relación a las iniciativas de e-participación, en particular para su estudio ulterior. Sin embargo, tiene el inconveniente de que dicho marco conceptual ha sido elaborado partiendo de la hipótesis de que son las autoridades políticas y las instituciones las que promueven proyectos de participación ciudadana, siendo así que muchas formas de e-participación surgen de abajo arriba (bottom-up), en cuyo caso el marco analítico que estamos comentando presenta algunas insuficiencias. El defecto principal, a nuestro modo de ver, consiste en no tener en cuenta el origen de las iniciativas en pro de la participación ciudadana. Para corregir esta insuficiencia habría que tener en cuenta los estudios de innovación distribuida de von Hippel (2005) y sus seguidores, buena parte de los cuales se centran en las innovaciones que surgen de los usuarios de los sistemas tecnológicos y de los servicios. En el caso de la e-participación, los estudios centrados en el uso de las herramientas que la posibilitan debería ser uno de los aspectos relevantes a tener en cuenta. Las tecnologías de participación que promueven las diversas agencias de la UE han de ir acompañadas por tecnologías socio-culturales que hagan accesible y fácil la participación mediante las tecnologías TIC. De no ser así, la ciudadanía no utilizará las TIC de e-participación, o no lo hará con suficiente soltura, frecuencia o fluidez. Como cualquier otra tecnología TIC, las herramientas que favorecen la e-participación aportan innovaciones tecnológicas, mayormente en el sector servicios. Por tanto, les resultan aplicables las teorías vigentes en los estudios de innovación, y en particular a los estudios de innovación social, de los que nos hemos ocupado en otro lugar ${ }^{21}$.

Ésta es una de las razones por las que pensamos que para promover la e-participación es preciso realizar previamen- 
te estudios empíricos sobre el uso de las TIC por parte de la ciudadanía, en particular cuando se utilizan para promover iniciativas ciudadanas o para protestar por algún producto o servicio deficiente.

\section{Participación electrónica y Agenda Digital EUROPEA}

En agosto de 2010 la Comisión Europea ha aprobado un nuevo programa para impulsar la sociedad de la información y las TIC, la Digital Agenda for Europe, que sustituye al programa i2010 y define otra estrategia y nuevos objetivos, en este caso para el año 2020. En el momento en que se escribe este artículo, no se conocen las acciones que lo implementarán, ni tampoco el posible giro en las acciones de e-participación que la Agenda Digital va a traer consigo. Por tanto, para terminar esta contribución haremos unas breves consideraciones en torno al nuevo documento de la $C E$, en el que se vuelve a insistir, ante todo, en la necesidad de crear un mercado común europeo en el espacio electrónico. Las líneas estratégicas son siete, pero la primera y la que ocupa más páginas, con diferencia, es el digital single market. Por tanto, el espacio europeo único de la información (SEIS) vuelve a ser concebido ante todo como un espacio donde ha de desarrollarse un mercado común, si bien la responsabilidad de impulsarlo y consolidarlo les corresponde en gran medida a los Estados. En conjunto los problemas estratégicos a afrontar son los siguientes ${ }^{22}$ :

a) Fragmentación del mercado digital. Se propone la creación de un mercado común digital, eliminando las barreras jurídicas y de otro tipo que todavía dificultan el funcionamiento de los mercados en el espacio electrónico europeo (SEIS).

b) Falta de interoperabilidad entre las distintas redes y plataformas que dan acceso al espacio electrónico ${ }^{23}$.

c) Aparición del cibercrimen y pérdida de confianza en la seguridad de las redes.

d) Falta de inversión en las redes.

e) Esfuerzos insuficientes de investigación e innovación en el sector TIC.

f) Carencias en la alfabetización digital y en la adquisición de destrezas para que la ciudadanía pueda operar en el espacio electrónico.

g) Oportunidades perdidas al afrontar desafíos sociales.
Comentaremos únicamente las dos últimas líneas estratégicas, porque en ellas se encuadrarán en un futuro las posibles acciones de fomento de la e-participación. Sin embargo, conviene señalar que, al menos en este primer documento de la nueva estrategia europea (Agenda 2020), impulsar los mercados electrónicos es mucho más importante que promover el e-gobierno y la e-participación. Continuamente se habla de los consumidores $y$, de hecho, el término "participación" sólo aparece una vez en el texto, cuando se habla de la participación de las mujeres y se pretende promover "a higher participation of young women and women returners in the ICT workforce through the support for web-based training resources, game based eLearning and social networking"24. Es posible que, en el marco de la nueva Agenda Digital Europea, la UE dé un nuevo impulso a la e-participación, pero por el momento esa tendencia no se manifiesta.

En la sexta línea estratégica se retoman los principios de la inclusión digital, sobre todo en relación a los 150 millones de europeos (un 30\% de la población) que nunca ha usado Internet. En su mayor parte son personas con más de 65 años, así como parados y personas con escasos ingresos y poca formación. La mayoria de ellos carecen de habilidades para usar las TIC, pero también de motivación, en muchos casos. También se indica que para 2015 puede haber 700.000 puestos de trabajo en el sector TIC que podrían no ser cubiertos por ciudadanos europeos, por falta de habilidades y destrezas. En suma, la sexta línea estratégica se centra en la educación y en la formación, con el fin de disminuir la exclusión y la brecha digital, pero no en la e-participación vinculada a la gobernanza y a la toma de decisiones, como ha sido entendida tradicionalmente.

En cuanto a la séptima línea estratégica, se centra en cuestiones relacionadas con el bienestar social, tales como el uso de las TIC en la lucha contra el recalentamiento del planeta, la e-salud y la diversidad cultural. También hay una cuarta línea de acción dedicada al gobierno electrónico, basada en un dato importante: sólo un 38\% de los usuarios de Internet acceden a servicios de gobierno electrónico, mientras que un $72 \%$ sí utiliza las TIC para asuntos de negocios. Además, se subraya que hay muy pocos servicios de gobierno electrónico supranacionales ${ }^{25}$ : cada uno de los Estados ha desarrollado sus propias herramientas de e-gobierno para su ámbito territorial, sin pensar en el conjunto de Europa. Pero también en este caso se insiste en que hay que tener en cuenta "the single market dimension of eGovernment" ${ }^{26}$, es decir, se 
piensa en la dimensión económica del desarrollo de dichas tecnologías y servicios, más que en su dimensión política y social. La CE comenta que va a desarrollar un plan ambicioso denominado eCommission 2011-2015 Action Plan, por lo que habrá que esperar a su publicación e implementación para sacar conclusiones definitivas, pero la impresión inicial es que la nueva Agenda Digital Europea no parece muy sensible a las cuestiones de e-participación, por lo que pudiera producirse una cierta regresión en este sentido.

De hecho, los autores del Study and Supply of Services on the Development of eParticipation in the $E U$ ya advirtieron que habian surgido críticas a la e-participación, así como algunas dudas sobre su eficacia. Coleman, Macintosh y Schneeberger (2008) señalaron que hay pocos estudios sobre la efectividad de la e-participación, así como de los procedimientos para evaluarla. Lippa y otros (2008) mostraron que la evaluación de la iniciativa de e-participación es inseparable del debate sobre los conceptos de e-participación y e-gobierno. También ha habido autores que han Ilamado la atención sobre los altos costes de las iniciativas de e-participación, sobre todo en relación a los resultados. En suma, cabe decir que el concepto de e-participación está siendo sometido a revisión en la UE, sobre todo cuando se le vincula a cuestiones de gobernanza. Hay un amplio consenso a favor de la e-inclusión y del e-gobierno, pero este último sólo se ha desarrollado en los distintos Estados, como la propia e-participación, sin haber alcanzado un nivel propiamente europeo.
A nuestro entender, y como ya hemos expuesto en publicaciones anteriores ${ }^{27}$, la emergencia del espacio electrónico implica cambios estructurales en la propia organización de los sistemas democráticos, debido a que la noción de démos ha estado vinculada tradicionalmente al territorio, mientras que en la sociedad red cabe hablar de una pluralidad de démoi interrelacionados a través de las redes, y por ende de un démos-red. Los problemas que hasta ahora ha tenido la UE en relación al gobierno electrónico y la participación electrónica ilustran bien estos problemas, porque todavía no cabe hablar de una e-ciudadanía, a no ser como un proyecto de futuro o como un ideal regulativo. Por el momento, los ciudadanos europeos son considerados como consumidores en el Single European Information Space (SEIS), en el que se pretende ante todo crear un mercado común dinámico y vibrante, que pueda competir con otras regiones del espacio electrónico global. Queda bastante tiempo hasta que las personas sean consideradas como ciudadanas en el SEIS, y no como consumidoras, trabajadoras o posibles clientes. La fragmentación de la UE en Estados sigue existiendo, así como la fragmentación del espacio electrónico europeo en diversos subespacios, sin que los programas eEurope e i2010 hayan satisfecho sus objetivos últimos. Falta por ver lo que ocurrirá en los próximos diez años con la Agenda Digital Europea 2020, pero los primeros documentos al respecto no parecen muy prometedores por lo que a la e-participación respecta.

\section{NOTAS}

1 Declaración de Ginebra, diciembre 2003, §4.

2 "Working together for growth and jobs: A new start for the Lisbon Strategy", COM(2005), 24.

3 "i2010: Una sociedad de la información para el crecimiento y el empleo", COM(2005) 229.

4 COM 2005, 229 de la Comisión Europea, fechada el 1 de junio de 2005 , pp. 3-4.

5 Otro tanto sucede en la Digital Agenda for Europe, aprobada en mayo de 2010 por la CE. En este artículo apenas nos ocuparemos de este nuevo plan, porque acaba de ser aprobado y todavía no ha sido implementado mediante acciones concretas.

$6 \operatorname{COM}(2005), 229$, p. 4.

7 lbid., p. 5.

8 lbid., p. 10.

9 Piénsese en el Plan D (Democracy, Dialogue and Debate), que fue aprobado ese mismo año.

10 COM (2007) 694 final, noviembre de 2007.

11 X. Barandiaran (ed.), Participación ciudadana institucional, San Sebastián, DFG, diciembre 2009, p. 77.

12 La literatura al respecto es abundante. Véase, por ejemplo, López Cerezo, Méndez Sanz y Todt 1998.
Aceptado: 3 de febrero de 2012

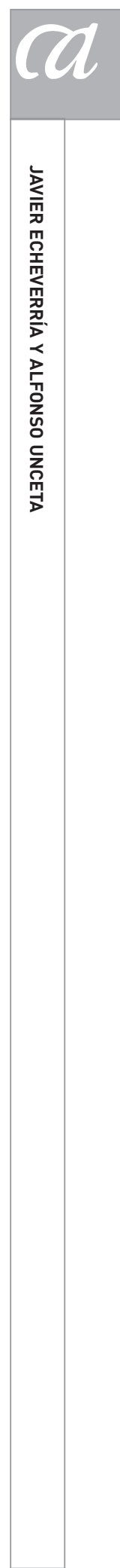


$13 \operatorname{COM(2007)} 694$ final, p. 7.

14 lbid., p. 8.

15 Sobre la noción del tercer entorno, ver J. Echeverría 1999.

16 La Declaración de Riga fue aprobada el 11 de junio de 2006 con ocasión de una conferencia de ministros sobre "ICT for an inclusive society". Los objetivos que se aprobaron se referian a un envejecimiento activo en el uso de las TIC, la brecha digital geográfica en Europa, la accesibilidad, la alfabetización digital, la diversidad cultural y el eGobierno inclusivo. Ver el portal de participación en la UE en islab/uom.gr/eP/, donde están este documento y otros muchos.

17 Ibid., p. 4.

18 Ver $\operatorname{COM(2010)~} 245$ final, p. 6.

19 El documento base sobre la gobernanza en la UE es COM(2001), 428 final.

20 Otro factor que se tiene muy en cuenta en dicho Study, ya desde la introducción (p. 4), es el de la candidatura de Obama a la Presidencia de los EEUU y el apoyo que tuvo en Internet y en las redes sociales. Este ejemplo es uno de los paradigmas recientes de e-participación en el espacio electrónico. Manuel Castells también le ha dedicado atención en su reciente libro Comunicación y Poder (2009, pp. 473-529.).

21 Ver Gurrutxaga y Echeverría, 2010.

$22 \operatorname{COM}(2010), 245$ final, p. 7.

23 Las plataformas que se tienen en cuenta explícitamente son las siguientes: "smartphone, tablet, personal computer, digital radio and highdefinition television" (Ibid., p. 4). Por tanto, el criterio multiplataforma del plan i2010 puede darse por consolidado.

24 Ibid., p. 26.
25 Ibid., pp. 31-32.

26 lbid.

27 Ver, por ejemplo, Echeverría 2000.

\section{BIBLIOGRAFÍA}

Barandiarán, X. (ed.) (2009): Participación ciudadana institucional, San Sebastián, DFG.

Castells, M. (1996-98): La era de la información, 3 vols., Madrid, Alianza.

Castells, M. (2009): Comunicación y poder, Madrid, Alianza.

Coleman, S., Macintosh, A. y Schneeberger, A. (2008): eParticipation Research Direction based on barriers, challenges and need, Demo-net D12.3, available at www.demo-net.org.

Council of Europe (2009): Recommendation on Electronic democracy, CM/ $\operatorname{Rec}(2009) 1$.

Cuevas, A. (2008): "Conocimiento científico, ciudadanía y democracia", Revista Iberoamericana de Ciencia, Tecnología y Sociedad, 10:4, pp. 67-83.

EC (European Commission) (2001): European governance, A White Paper, $\operatorname{COM(2001):~} 428$ final, Bruselas, UE.

EC (European Commission) (2002): eEurope: una sociedad de la información para todos, COM(2002), 263, Bruselas, UE.

EC (European Commission) (2005a): Working together for growth and jobs: $A$ new start for the Lisbon Strategy, COM(2005) 24, Bruselas, UE.

EC (European Commission) (2005b): 2010 : Una sociedad de la información para el crecimiento y el empleo, COM(2005) 229, Bruselas, UE.

EC (European Commission) (2007): Iniciativa Europea i2010 para la inclusión digital: "participar en la sociedad de la información", COM (2007) 694 final, Bruselas, UE.
EC (European Commission) (2009): "European eParticipation, Summary Report", November 2009, prepared by the Danish Technological Institute, Leeds University and University of Macedonia.

EC (European Commission) (2010): A digital Agenda for Europe, COM(2010) 245 final/2, Bruselas, UE.

Echeverría, J. (1994): Telépolis, Barcelona, Destino, 1994.

Echeverría, J. (1999): Los Señores del Aire: Telépolis y el Tercer Entorno, Barcelona, Destino.

Echeverría, J. (2000): "Democracia y sociedad de la información", Isegoría 22, pp. 37-57.

Gurrutxaga, A. y Echeverria, J. (2010): La luz de la luciérnaga. Diálogos de innovación social, Bilbao, ASCIDE.

Lippa, B. et al. (2008): eParticipation EvaIuation and Impact, Demo-net 13.3, available at www.demo-net.org.

López Cerezo, J. A.; Méndez Sanz, J. A. y Todt, O. (1998): "Participación Pública en Política Tecnológica", Arbor, CLIX, n. ${ }^{\circ} 627$, pp. 279-308.

ONU, Informe final de la Fase de Ginebra de la Cumbre Mundial sobre la Sociedad de Ia Información, Documento WSIS-03/ GENEVA/9(Rev.1)-S, diciembre 2003.

ONU, Programa de Acciones de Túnez para la Sociedad de la Información, Documento WSIS-05/TUNIS/D0C/6(Rev.1)S, 2005.

ONU, Compromiso de Túnez, Documento WSIS-05/TUNIS/D0C/7-S, noviembre 2005.

Rifkin, J. (2000): La Era del Acceso, BarceIona, Paidós.

Smith, S.; Macintosh, A. y Millard, J. (2008): European eParticipation. Study and supply of services on the development of eParticipation in the EU, Bruselas, European Commision.

Von Hippel (2005): Democratizing Innovation, MIT Press, Mass. 